\title{
Assessing the Dosimetric Consequence of Inter-fractional Setup Shifts on Helical TomoTherapy Plans with Independent Dose Calculation
}

\author{
Weihong He, Luis A Vazquez Q, Eric Dzintars and Nikos Papanikolaou*
}

Cancer Therapy and Research Center, Department of Radiation Oncology, University of Texas Health Science Center at San Antonio, TX, USA

\begin{abstract}
This work studied on dosimetric impact due to inter-fractional uncertainties for one hundred patients from five different treatment sites (30 prostate, 26 head \& neck, 18 lung, 17 pelvis, and 9 brain patients) for Tomotherapy modality. Daily setup shifts were quantified and grouped into systematic (mean daily setup shifts) and random shifts (fractionbased shifts with corresponding systematic shift subtraction). Both systematic and random shifts were incorporated into in-house independent point dose calculation software, MU-Tomo, to separately evaluate the systematic and random dosimetric variations. Systematic dosimetric variations showed large dose deviation, with the largest difference at $-10.02 \%$ compared to the planned dose and $3 \%$ standard deviation. Mean random dosimetric variations showed relatively small dose deviation with the largest at $-5.65 \%$ compared to the planned dose and $1.9 \%$ standard deviation. Furthermore, different treatment sites were sorted into the head \& neck and brain group, and the body group including lung, pelvis, and prostate cancers. According to ANOVA analyses, random dosimetric variations were found significantly different between patients treated at the same treatment site, while systematic dosimetric variations were significantly different between the head \& neck and brain group and the body group. No significant differences were discovered among specific patients for systematic dosimetric variations, and no significant differences were observed within each of the two groups for random dosimetric variations. Dosimetric consequences are not significantly correlated with treatment fraction number according to the Pearson correlation analysis. By comparing doses without any shift against those with the random shift, overall dosimetric impacts to each patient were found to be very small with the mean value $-0.0053 \%$ and standard deviation of $1.11 \%$. Ninety-nine percentage of the averaged variation results were within $3.5 \%$. This implies that overall dosimetric impact from random variations is small; instead, dosimetric impact is more affected by systematic shifts.
\end{abstract}

Keywords: Helical tomotherapy; Independent dose calculation; Daily setup shift; Inter-fractional study

\section{Introduction}

Due to the highly conformal nature, intensity modulated radiation therapy (IMRT) delivery requires high precision and accuracy in target localization. Incorrect target localization may induce dose delivery errors. Image guided radiation therapy (IGRT) has thus become a popular approach to eliminate the geometric uncertainties (Mackie et al., 2003; Perkins et al., 2006; Sheng et al., 2008; Stutzel et al., 2008). With on-board megavoltage computed tomography (MVCT), helical tomotherapy (HT) (TomoTherapy, Inc., Madison, WI, USA) is able to plan and deliver conformal dose distribution to the target volume with fan beam radiation (Mackie, 2006). IMRT plans from tomotherapy are generally produced with fifty-one projections per helical rotation. During plan optimization, the control to the binary multi-leaf collimator (MLC) yields the sinogram to direct the plan delivery based on the target tumor setting. Helical delivery along sinogram determines that tomotherapy is patient sensitive, especially to the target positioning, because equal and opposite shifts do not necessarily because equal and opposite dose distribution even for the same sinogram patterns at symmetric positions. Careful management of not only the geometric uncertainties but also the dosimetric impacts from geometric uncertainties is of great interest.

In clinical practice, patient positioning uncertainties are mainly generated by machine misalignment, intra-fraction and inter-fraction motions from the patient. Detrimental dose impacts to the target volume as well as to the normal tissues or organs at risk are the primary concern. Dosimetric effect due to machine misalignment has been investigated by different groups for various modalities including tomotherapy (Su et al., 2008) and Linac (Low et al., 1997;
Xing et al., 2000) machines. From these studies, it was shown that machine misalignment in clinical practice could yield significant dose differences, and that damage to organs at risk might be profound. To take advantage of the IMRT technique in radiation therapy, it is important to minimize dosimetric errors with accurate machine alignment and proper patient setup. Intra-fractional motion of patients could induce even worse detrimental dose distributions than the machine misalignment. Patient activities, such as respiration and motion, cause difficulties during treatment (Keall et al., 2006; Sawant et al., 2008; Lefkopoulos et al., 2007). For helical tomotherapy, real time motion adaptive optimization (Lu et al., 2009) and the impact of intra-fraction motion (Ngwa et al., 2009) are among the major representatives to improve dose distributions caused by intrafractional motion.

For inter-fractional motion, the MVCT provides a powerful tool to study the inter-fractional properties of the patient activities and the associated dosimetric effect. MVCT is utilized for daily setup

*Corresponding author: Dr. Nikos Papanikolaou, 7979 Wurzbach Rd Ste 240 Cancer Therapy and Research Center, Department of Radiation Oncology, University of Texas Health Science Center at San Antonio, TX, USA, Tel: 210-450-1027; Fax: 210-616-5682; E-mail: shic@uthscsa.edu

Received July 08, 2010; Accepted September 01, 2010; Published September 01, 2010

Citation: He W, Vazquez LA, Dzintars E, Papanikolaou N, Shi C (2010) Assessing the Dosimetric Consequence of Inter-fractional Setup Shifts on Helical TomoTherapy Plans with Independent Dose Calculation. J Cancer Sci Ther 2: 136 144. doi:10.4172/1948-5956.1000039

Copyright: (C) $2010 \mathrm{He} \mathrm{W}$, et al. This is an open-access article distributed under the terms of the Creative Commons Attribution License, which permits unrestricted use, distribution, and reproduction in any medium, provided the original author and source are credited. 
registration. Tomotherapy treatment plans are based on the kilovoltage computed tomography $(\mathrm{kVCT})$ for each patient. The original kVCT is stored in the treatment station and online MVCT scans for the same patient will be registered to the original kVCT images. Tomotherapy provides auto-adjustments based on the registration results in most directions, such as superior-inferior (IEC-Y), anteriorposterior (IEC-Z), and roll deviations, but not in the lateral direction (IEC-X). Manual adjustment is required in the IEC-X direction. Although there have been many studies reporting on the effect of patient setup shifts for conformal or IMRT techniques from different treatment sites (Zhou et al., 2010; Fiorino et al., 2007; Siebers et al., 2005; Manning et al., 2001; Fu et al., 2006; Landoni et al., 2006; Balter et al., 2005), most patient setup shift studies in helical tomotherapy focused on the MVCT itself with novel registration strategies or statistics (Yan et al., 2005; Broggi et al., 2007, 2009; Lee et al., 2008; Lin et al., 2009; Rivest et al., 2009; Li et al., 2007). A few studies reported on dosimetric consequences with the MVCT setup shifts for patients with cancer from prostate (Kalz et al., 2009) and breast (Goddu et al., 2009), where dose re-calculations implemented the same tomotherapy treatment or adaptive planning system as the original plan.

In our previous study, the inter-fractional daily setup shifts with more than a hundred patient cases were reported with systematic analyses (Lin et al., 2009). However, dosimetric consequence from these inter-fractional shifts was not reported. Therefore, the goal of this study is to investigate the dosimetric consequence of inter-fractional setup shifts on helical tomotherapy plans. Previous studies have shown that dosimetric discrepancies were negligible when the gantry rotation is within $\pm 2^{\circ}$ (Su et al., 2008, Fu et al., 2006) and the rotation uncertainties are less than $\pm 2^{\circ}$. Therefore, rotational effect is not examined in this study. The daily setup shifts were quantified, grouped, and incorporated into our independent dose calculation software, $M U$-Tomo. The mean daily setup shift is defined as the systematic shift and the fraction-based shift with corresponding systematic shift subtraction is defined as the random shift. Both systematic and random shifts were incorporated into our in-house independent point dose calculation software, $M U$-Tomo, to separately evaluate the systematic and random dosimetric variations. Systematic dosimetric variations and random dosimetric variations were then analyzed.

\section{Materials and Methods}

\section{Patient selection, planning, and shift quantification}

One hundred cancer patients from multiple treatment sites managed by helical tomotherapy were randomly selected for this study. In the selected samples, each patient was assigned with one treatment plan with individual kVCT images scanned by a GE light speed 4-slice CT scanner (General Electric Medical Systems, Milwaukee, WI, USA). There are a total of thirty prostate cases, twenty six head \& neck cases, eighteen lung cases, seventeen pelvis cases, and nine brain cases evaluated from the past one and one half year.

Treatment plans for each patient were generated with the corresponding kVCT image. In our center, kVCT images are initially imported to the Pinnacle ${ }^{3}$ treatment planning system (Pinnacle $^{3}$ TPS, Philips Medical Systems, Andover, MA, USA). To prepare for tomotherapy treatment planning, patients were contoured with the planning target volume (PTV) and organs at risk. Contoured CT images were then transferred to the tomotherapy treatment planning station by DICOM network. For all patients in this study, they were treated with the $2.5 \mathrm{~cm}$ jaw setting. The goal of the tomotherapy planning system is to constrain the plan dose to the target volume. Our treatment plans were optimized according to treatment site specific optimization criteria. Final dose distributions were created, and delivery procedures were used for treatment deliveries. The beam projections and delivery information were utilized to create delivery quality assurance (DQA) plans. These DQA plans were generated on the Cheese phantom (a cylindrical phantom with radius $15 \mathrm{~cm}$ and length $18 \mathrm{~cm}$ ). From the DQA plans, a random point inside the tumor setting was selected and the planned point dose was retrieved, which would be compared with our independent dose calculation at the same point on the same Cheese phantom using our in-house second check software, $M U$-Tomo.

With pretreatment MVCT scans for each treatment fraction of each patient, daily setup shifts were obtained during kVCT and MVCT registrations. Shifts were classified into IEC-X, IEC-Y, and IEC- $Z$ directions and quantified as the software input. The raw interfractional daily setup shifts were incorporated with the selected point coordinates from the DQA planning step, therefore the Cheese Phantom in MU-Tomo was shifted accordingly and the dose was recalculated to examine the dosimetric impact due to inter-fractional patient setup shifts.

\section{Independent Dose Calculation Method: $M U$-Tomo}

Based on the patient information and dosimetric functions, such as off-axis ratios along the IEC-X and IEC-Y directions (OARX and OARy), tissue phantom ratio (TPR), and output function (Scp), an independent dose calculation software for helical tomotherapy has been developed. The correction-based software is named $M U$ Tomo and performs independent point dose calculations. Dosimetric functions are retrieved from our tomotherapy machine commissioning data. Patient information can be separated into two parts: userdetermined information and planning-determined information. Userdetermined information includes the point of interest coordinates inside the tumor target and the planned dose of the point of interest from the DQA plan. The Tomotherapy machine provides two laser systems: green lasers for benchmark purposes and red lasers for target localization purposes. In the Tomotherapy treatment planning station, the green and red lasers are used to identify the point of interest for dose calculation. In our study, coordinates from both laser cross-points were recorded and the relative differences from the green laser point to the red laser point were calculated in the IEC-X, IEC-Y, and IEC-Z, individually. The relative differences in all three dimensions served as the input coordinates for the point of interest. The planned dose of the point of interest is compared against the $M U$ Tomo calculated dose for the same point of interest. The independent dose calculation is executed and the second check result is concluded. Planning-determined information includes all dynamics for the couch movement, MLC movement, and gantry movement. During treatment planning optimization, delivery dynamics are all determined and stored. The archived patient Extensible Markup Language (XML) file provides the dynamics for $M U$-Tomo dose calculation.

(Figure 1 here: The MU-Tomo second check software working platform.)

$$
D_{p}=\dot{D}_{0} \times \sum_{i=1}^{N_{\text {proj }}}\left(\frac{85}{S P D_{i}}\right)^{2} \times O A R_{X}\left(X_{i}\right) \times \sum_{j=1}^{N_{\text {seg, }} \cdot t}\left\{t_{i j} \times S_{c p, j} \times T P R_{j}\left(d_{i}\right) \times O A R_{y, j}\left(Y_{i}, d_{i}\right)\right\}
$$

The dose calculation formula [1] used in our second check software was proposed by Gibbons et al. (2009). It shows how the dose at a point $\mathrm{P}$ is calculated based on the information entered into $M U$ - 


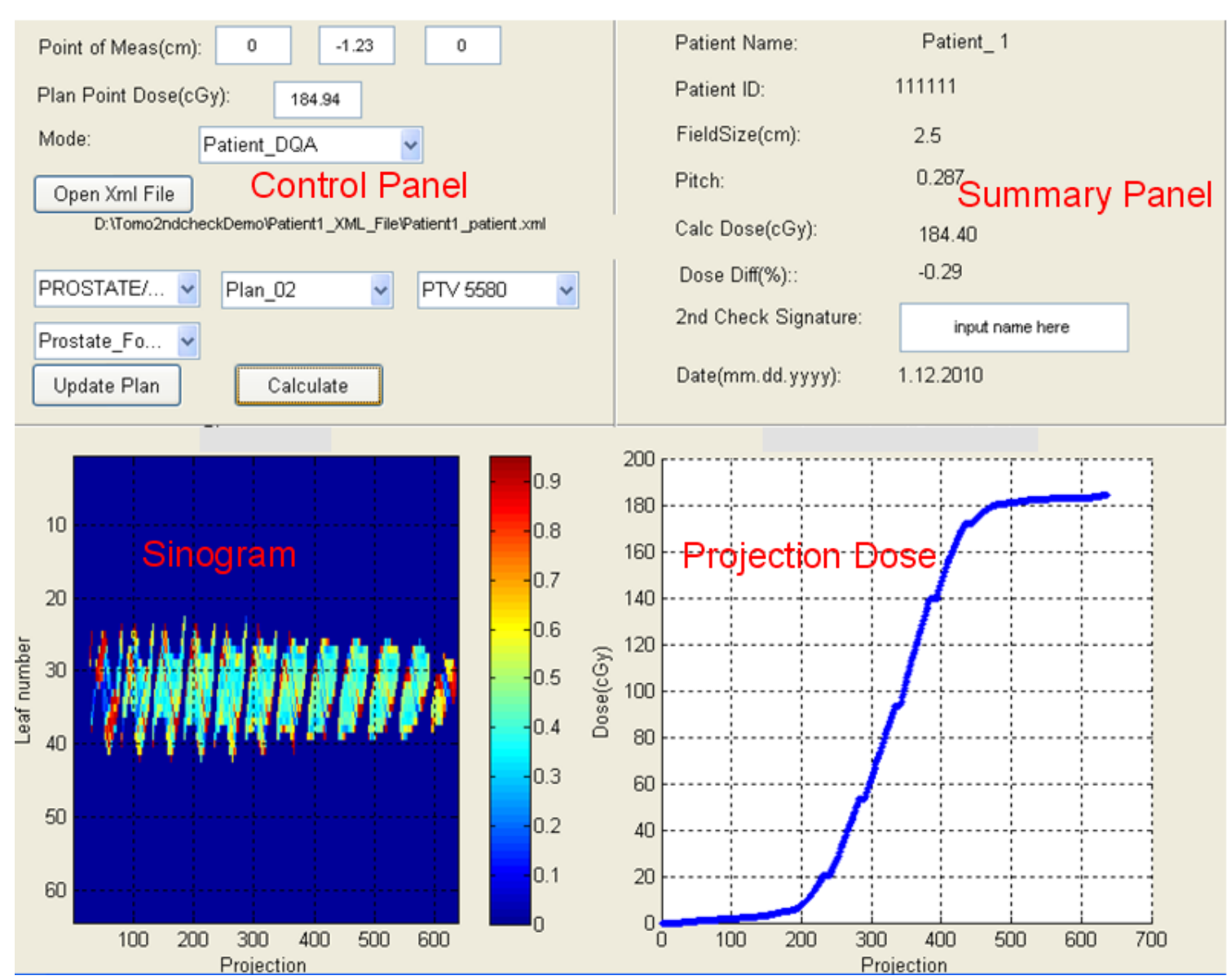

Figure 1: The MU-Tomo second check software working platform.

Tomo. Dosimetric functions are essential for this correction- based software. Dosimetric functions from commissioning data represent real measurement-based characteristics for a specific tomotherapy machine. Off-axis ratios laterally (OARx) and longitudinally (OARy), tissue-phantom ratios (TPR) calculated from percent depth dose (PDD), and output functions (Scp) measured from different field sizes are all embedded as a kernel of the MU-Tomo software. The dose calculation integrates those commissioning data, patient information, and beam dynamics. $D_{0}$ is the dose rate measured at depth $10 \mathrm{~cm}$ in solid water and field size $40 \times 5 \mathrm{~cm}^{2}$ with SAD (source to axis distance) $=85 \mathrm{~cm}$. SPD is the source to point of calculation distance. For helical Tomo Therapy treatment, radiation beams are irradiated with 51 projections during each gantry rotation. For each projection, there is a specific SPD because of the geometrical change. Both OARx and OARy were normalized at the maximum value. OARx and OARy show different profile characteristics for Tomotherapy modality. OARx profiles measured from different open field widths are stable at the normalized depth $10 \mathrm{~cm}$. OARy profiles exhibit profound variations from different depths even for the same open field width. In the development, OARx profiles measured from the depth of $10 \mathrm{~cm}$ were utilized; and OARy profiles from depth $1.5 \mathrm{~cm}$, $5 \mathrm{~cm}, 10 \mathrm{~cm}, 15 \mathrm{~cm}$, and $20 \mathrm{~cm}$ were embedded in the software. $S c p, j$ is the output factor in the $j_{\text {th }}$ segmentation, and $t_{i j}$ is the delivery time in the $j$ th segmentation within the ith projection. Segmentation is based on the MLC sinogram file. For a general Tomo Therapy IMRT plan, sinogram file provides the leaf movement and opening time for each projection. The leaf opening times along 32 pairs of leaves are normally un-symmetric. MU-Tomo symmetrically averages the leaf opening time around the central leaf right above the point of calculation, and new leaf sequence is determined from the largest open leaf field to the central leaf with evenly distributed time pattern, where the central leaf is directly above the point of calculation. An example of calculation is shown in Figure 1. The working platform in Figure 1 illustrates the beam dynamics at the lower-left corner with a sinogram map. The colored intensities in the sinogram represent the multi-leaf collimator (MLC) movement and opening time. MLC in helical tomotherapy follows binary rules: non-zero intensity means open MLC and duration, while zero intensity means closed MLC. Controls to MLCs are projection-based. Depending on the size and localization of tumor target setting, the sinogram map may contain a different number of projections. Projections are dynamically synchronized with couch movement and a certain pitch. When the point of interest and patient archived document are determined, the planned point dose, main plan name, QA plan name, target tumor, and treatment site will show up for users to select. During dose calculation in $M U$-Tomo, the point of interest is dynamically related to the beam radiation controlled by the sinogram and corresponding dosimetric functions. Therefore, the procedure of dose calculation in $M U$-Tomo is the accumulation of dose depositions from projection to projection. This is illustrated in the lower-right plot of Figure 1. The dose calculation result is displayed in the summary panel of Figure 1. Compared to the planned point dose, a dose difference 
can be concluded. This whole calculation procedure is performed automatically.

\section{Patient setup shift}

A total of thirty prostate cancer cases, twenty six head \& neck cancer cases, eighteen lung cancer cases, seventeen pelvis cancer cases, and nine brain cancer cases were analyzed. The mean daily setup shift for each patient was calculated as the systematic shift. The mean systematic shift of all patients with the same treatment site is summarized in Table 1 along with standard deviation, minimum, and maximum. The largest mean systematic shift occurred in IEC-Z among the three dimensions. The maximum systematic shift in the IEC-X direction was up to $12 \mathrm{~mm}$ in a pelvis patient, the maximum systematic shift in the IEC-Y direction was up to $20 \mathrm{~mm}$ in a lung patient, and the maximum systematic shift in the IEC-Z direction was up to $17 \mathrm{~mm}$ in pelvis, prostate, and lung patients. The IEC-Z direction was observed to have the largest mean systematic shift within each treatment site. Since the random shift for each fraction of each patient was calculated from the patient's systematic shift and raw daily setup shift, the mean of the random shifts over all treatment fractions is always zero. Each patient had a standard deviation from random shifts along treatment course. Therefore, only the mean standard deviations of random shifts are summarized in Table 2 for patients from different treatment sites.

(Table 1 here. Mean systematic shifts with standard deviations (SD) for different treatment sites. Positive and negative signs denote the coordinate directions of the Tomotherapy system.)

(Table 2 here. Mean standard deviations (mean SD), min, and max of random shifts for patients from different treatment sites).

\section{Independent dose calculation validation}

Dose calculation and validation has been performed for all selected patients. During our study, calculations typically finished within twenty seconds for each of the hundred patients using a computer with an Intel dual-core CPU@3 GHz processor and 3 GB RAM. To evaluate the dosimetric consequence due to inter-fractional shifts of helical tomotherapy patient daily setup, benchmark dose calculations are required from the independent dose calculation software. As the software performs a point dose calculation, the first task before validation is to select the point position and planned point dose for each patient.

For all patients, points were chosen within the target tumor setting region. The target tumor setting regions were positioned near the center of the cylindrical Cheese phantom during DQA procedures. By doing this, points were not necessarily located at the center of the phantom, but were always inside the phantom. In this status, the patient and phantom were treated as static to serve as a reference for the following daily setup shifts study. Planned point doses were obtained from the treatment planning station. Meanwhile, planned point doses for different patients varied depending on the treatment sites and point locations, but were all accumulated point doses for all fractions. Therefore, the planned point dose per fraction for every patient was calculated from the total dose and the specific treatment fraction number. With the relative coordinates of the point of interest, archived patient documents, and planned point dose, $M U$ Tomo performed independent dose calculation and presented the second check results as the reference dose differences. In this step, not only the reference point coordinates were selected, but also the reference dose differences were determined for all patients. The two elements served as the benchmark to the inter-fractional study.

\section{Inter-fractional Data Analyses}

In this study, each patient had a treatment plan with different treatment fraction numbers. Raw daily setup shifts data were collected for our analyses. The mean displacements for each patient over all corresponding fractions were calculated. The mean displacements for each patient represented the systematic inter-fractional shifts in the following independent dose calculation. Systematic shifts were separately incorporated into the point of interest coordinates in IEC-X, IEC-Y, and IEC-Z directions, so that MU-Tomo calculated the systematic dosimetric variation for each patient over all treatment fractions. Based on the mean displacements for each patient's overall treatment fractions, the specific subtractions from the raw daily patient setup shifts to the mean displacements were gained for each treatment fraction. These subtractions resulted in the random shifts for each fraction. The random shifts were incorporated into the point of interest coordinates in the IEC-X, IEC-Y, IEC-Z directions. MU-Tomo then calculates the random dosimetric variation for each treatment fraction of each patient. With the random dosimetric variation from each fraction, mean dose differences with standard deviations for each patient over the treatment fractions were calculated. Patients were classified into five groups according to treatment sites: prostate, head \& neck, lung, pelvis, and brain. Simple statistics with mean dose differences and standard deviations for each patient over the entire treatment course were reported into the five different groups. Furthermore, with the random dosimetric variation results, treatment

\begin{tabular}{|c|c|c|c|c|c|c|c|c|c|c|}
\hline \multirow{2}{*}{ Treatment site } & \multirow{2}{*}{ \# of Patients } & \multicolumn{3}{|l|}{ IEC-X(mm) } & \multicolumn{3}{|l|}{$\mathrm{IEC}-\mathrm{Y}(\mathrm{mm})$} & \multicolumn{3}{|l|}{$\mathrm{IEC}-\mathrm{Z}(\mathrm{mm})$} \\
\hline & & mean $\pm S D$ & $\min$ & $\max$ & mean $\pm S D$ & $\min$ & $\max$ & mean $\pm S D$ & $\min$ & $\max$ \\
\hline Prostate & 30 & $-2.9 \pm 3.1$ & -0.1 & -10.8 & $-2.3 \pm 3.1$ & 0 & -6.9 & $10.98 \pm 4.48$ & -0.1 & 16.9 \\
\hline $\mathrm{H} \& \mathrm{~N}$ & 26 & $-3.5 \pm 2.8$ & -0.1 & -7.9 & $-1.1 \pm 2.3$ & -0.3 & 3.9 & $6.09 \pm 2.23$ & 2.6 & 11.2 \\
\hline Lung & 18 & $-1.7 \pm 4.3$ & 0.1 & -11 & $-2.8 \pm 7.3$ & 0.1 & -20.6 & $4.72 \pm 8.11$ & -0.2 & 16.4 \\
\hline Pelvis & 17 & $-2.7 \pm 3.6$ & 0 & -12.2 & $-1.1 \pm 5.3$ & 0 & 15.3 & $9.06 \pm 4.88$ & 0 & 17.4 \\
\hline Brain & 9 & $-1.7 \pm 1.2$ & 0.3 & -3.8 & $-3.9 \pm 3.7$ & -0.6 & -11.7 & $4.81 \pm 2.16$ & 0.9 & 6.7 \\
\hline
\end{tabular}

Table 1: Mean systematic shifts with standard deviation (SD) for dif ferent treatment sites. Positive and negative signs denote the coor dinate directions of Tomotherapy system.

\begin{tabular}{|c|c|c|c|c|c|c|c|c|c|c|}
\hline \multirow{2}{*}{ Treatment site } & \multirow{2}{*}{ \# of Patients } & \multicolumn{3}{|l|}{ IEC-X(mm) } & \multicolumn{3}{|c|}{ IEC-Y(mm) } & \multicolumn{3}{|l|}{ IEC-Z(mm) } \\
\hline & & mean SD & $\min$ & $\max$ & mean SD & $\min$ & $\max$ & mean SD & $\min$ & $\max$ \\
\hline Prostate & 30 & 4.4 & 1.2 & 11.2 & 2.2 & 1.0 & 6.0 & 2.6 & 1.6 & 4.7 \\
\hline $\mathrm{H} \& \mathrm{~N}$ & 26 & 2.0 & 0 & 4.5 & 2.2 & 0 & 6.7 & 1.9 & 0 & 3.9 \\
\hline Lung & 18 & 3.1 & 1.0 & 7.0 & 4.2 & 1.3 & 7.4 & 4.2 & 1.8 & 9.9 \\
\hline Pelvis & 17 & 2.9 & 1.0 & 7.2 & 2.3 & 1.3 & 5.0 & 2.7 & 1.3 & 4.0 \\
\hline Brain & 9 & 1.6 & 1.0 & 4.5 & 3.2 & 1.2 & 8.7 & 1.2 & 0.7 & 1.9 \\
\hline
\end{tabular}

Table 2: Mean standard deviations (mean SD), min, and max of random shifts for patients from different treatment sites. 


\begin{tabular}{|c|c|c|c|c|c|c|c|}
\hline \multirow{2}{*}{ Treatment site } & \multirow{2}{*}{ \# of Patient } & \multicolumn{3}{|c|}{ MU-Tomo calculated result } & \multicolumn{3}{|l|}{ Planned Dose } \\
\hline & & Mean $\pm S D$ & Max & Min & Mean $\pm S D(c G y)$ & Max (cGy) & Min (cGy) \\
\hline Prostate & 30 & $0.47 \% \pm 3.83 \%$ & $3.83 \%$ & $-3.41 \%$ & $205.55 \pm 31.03$ & 293.81 & 147.76 \\
\hline $\mathrm{H} \& \mathrm{~N}$ & 26 & $0.14 \% \pm 2.65 \%$ & $4.76 \%$ & $-5.73 \%$ & $167.87 \pm 90.51$ & 497.20 & 81.86 \\
\hline Lung & 18 & $0.0 \% \pm 1.99 \%$ & $4.06 \%$ & $-3.34 \%$ & $186.06 \pm 42.79$ & 272.30 & 132.49 \\
\hline Pelvis & 17 & $-0.07 \% \pm 1.66 \%$ & $3.05 \%$ & $-2.94 \%$ & $210.69 \pm 33.71$ & 312.59 & 175.24 \\
\hline Brain & 9 & $0.37 \% \pm 2.40 \%$ & $5.66 \%$ & $-2.97 \%$ & $161.63 \pm 59.26$ & 304.10 & 125.00 \\
\hline
\end{tabular}

Table 3: MU-Tomo calculated dose differences compared with planned doses information. Dose information for each group of patients is summarized here with the mean value, standard deviation (SD), maximum and minimum dose deviation.
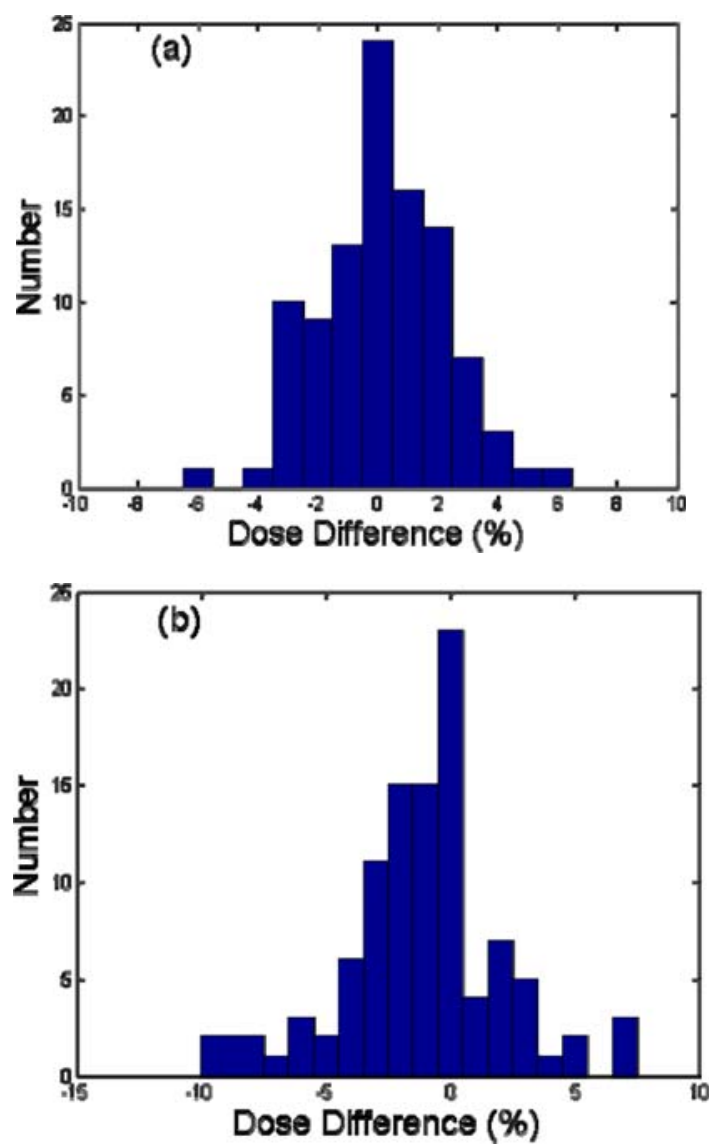

Figure 2: Both plots show the independent dose calculation result from 100 helical tomotherapy patient plans with MU-Tomo. (a) Second check results without shifts (non-shift dose differences). (b) Dose difference consequences with systematic inter-fraction setup shifts.

site specific dosimetric consequence results were reported with the mean dose differences, standard deviation, maximum deviation, and minimum deviation.

To test the relationships of dosimetric consequences (both systematic dosimetric variation and random dosimetric variation) among specific patients, among treatment sites, and between specific patients and fraction numbers, the results were analyzed with three statistical tests. To evaluate the patient specific dosimetric consequences due to inter-fractional setup shifts, the Kruskal-Wallis analysis of variance (ANOVA) test was performed to compare all the patients in each treatment site category with a confidence level of $95 \%$, which corresponds to the statistical test threshold $\alpha=0.05$. To evaluate the tumor site specific dosimetric consequences due to inter-fractional setup shifts, the ANOVA test was used with the same method by separating the treatment sites into two groups: the head
\& neck and brain group, and the body group including lung, pelvis, and prostate cancers. The two groups were classified according to whether the treatment site conducts apparent internal motion, because internal motion could also affect the daily setup shifts. The treatment site specific ANOVA tests were performed between the two groups and within each group. The third test was performed with the Pearson correlation statistical method to examine the relationship between treatment fraction numbers and dosimetric consequences within patients who had the same treatment site. For the first two statistical tests, $p$ values were calculated to determine the statistical significance. For the third test, correlation coefficients were derived to show the correlation relationship. For all the statistical tests, both systematic variation and random variation of the dosimetric consequences were examined. Furthermore, to test the overall dosimetric impact, Kruskal-Wallis analysis was performed between the systematic dosimetric variations and mean random dosimetric variations for all patients.

The effect of the overall dosimetric impact to each patient was evaluated by analyzing the benchmark dose difference. The benchmark dose difference is calculated by subtracting the reference dose difference calculated during the software validation step from the mean random variation of dosimetric consequence from all treatment fractions of each patient. Since the random shift for each treatment fraction has already been corrected from the systematic shift, the benchmark dose difference is actually the result of comparing doses with and without the daily setup shifts after systematic correction. By doing this analysis, not only the dosimetric impact of inter-fractional motion, but also the utility of IGRT have been studied.

\section{Results}

\section{Independent Dose Calculation Validation with MU-Tomo}

$M U$-Tomo calculated the dose for the corresponding point independently. The calculated point dose was compared to the planned dose to conclude the dose difference in percentage for each patient. Table 3 shows the sorted MU-Tomo calculated dose difference results compared to the planned dose information from the five treatment sites. Dose information for each group of patients is listed with the mean value, standard deviation, maximum and minimum dose deviation. Ninety eight of the one hundred patient's second check results fall within $\pm 5 \%$. For the selected one hundred patient results, the mean dose difference between planned and independently calculated dose was $0.20 \%$ with a standard deviation of $2.06 \%$. Figure 2 (a) shows the comprehensive dose difference histogram between planned and calculated doses in percentage. Furthermore, Pearson analysis between the planned doses and $M U$ Tomo calculated dose differences from all patients was conducted and concluded $\rho=-0.078$, which means $M U$-Tomo calculated dose differences are not correlated with planned doses. Kruskal-Wallis ANOVA analysis was performed to the MU-Tomo calculated dose differences between difference treatment sites and found that 
$\mathrm{p}=0.87$, which means dose differences are not correlated with treatment sites. Results show that $M U$-Tomo is a robust tool and can perform the independent dose verification with acceptable tolerance $\pm 5 \%$ recommended by ICRU Report 29 (1978).

(Table 3 here. $M U$-Tomo calculated dose differences compared with planned doses information. Dose information for each group of patients is summarized here with the mean value, standard deviation, maximum and minimum dose deviation.)

(Figure 2 here. Both plots show the independent dose calculation result from 100 helical tomotherapy patient plans with MU-Tomo. (a) Second check results without shifts (non-shift dose differences). (b) Dose difference consequences with systematic inter-fractional setup shifts).

\section{Inter-fractional studies}

All dose calculations and comparisons were performed by $M U$ Tomo software. Systematic dosimetric variations from all patients were calculated and illustrated in Figure 2 (b), as a comparison to the reference of validation result. Systematic dosimetric variation for each patient results from the systematic shift during the whole treatment course. The distribution of systematic dosimetric variations from the one hundred patients was much wider than the reference of validation result. The maximum dose difference was $7.31 \%$ and the minimum dose difference was $-10.02 \%$ for the systematic dosimetric variations. The standard deviation of systematic dosimetric variations was $3 \%$.

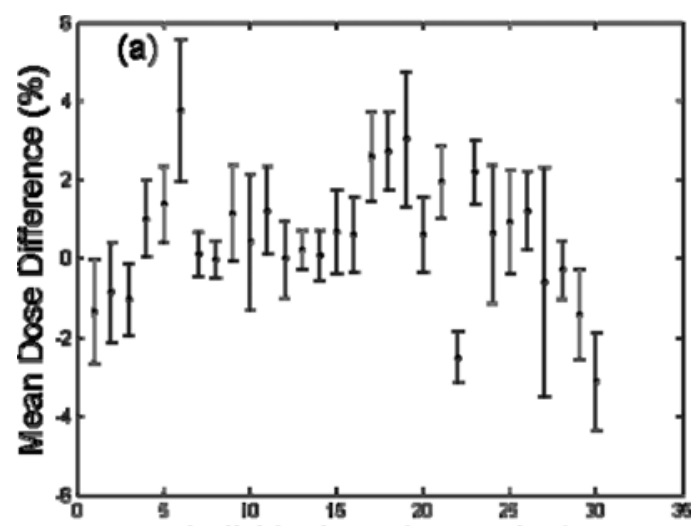

Indlvidual prostate patlent
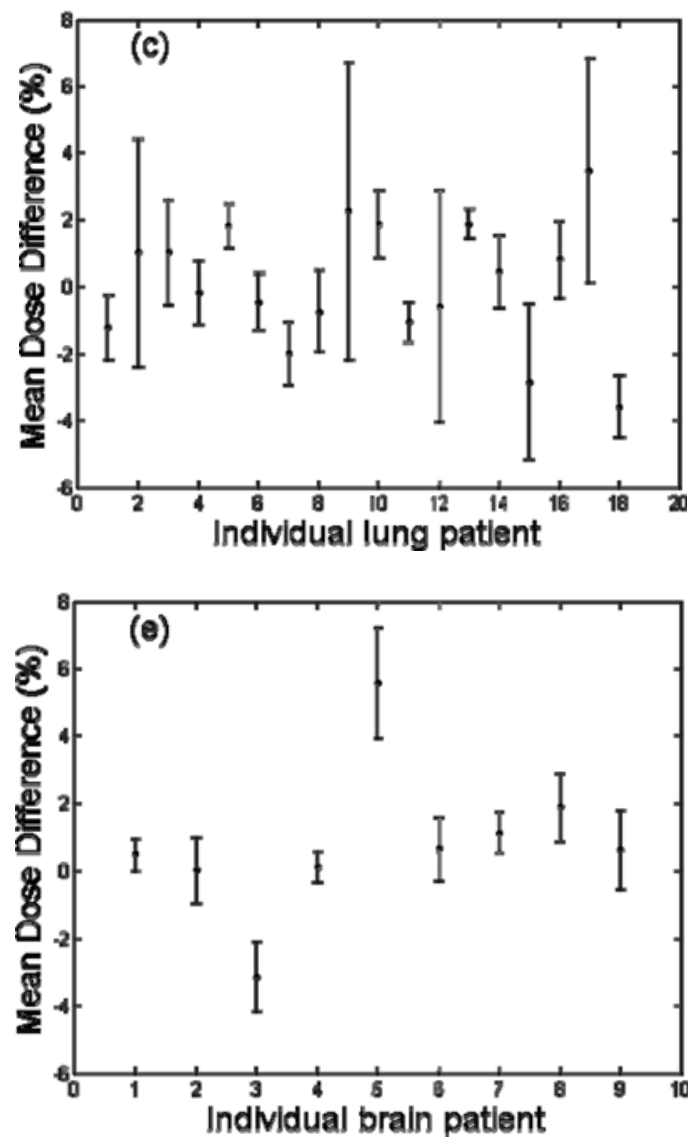

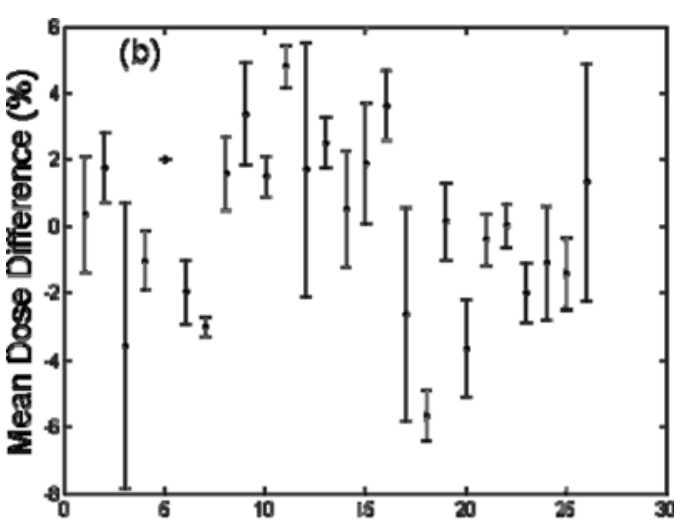

Indlvidual head\&neck patient

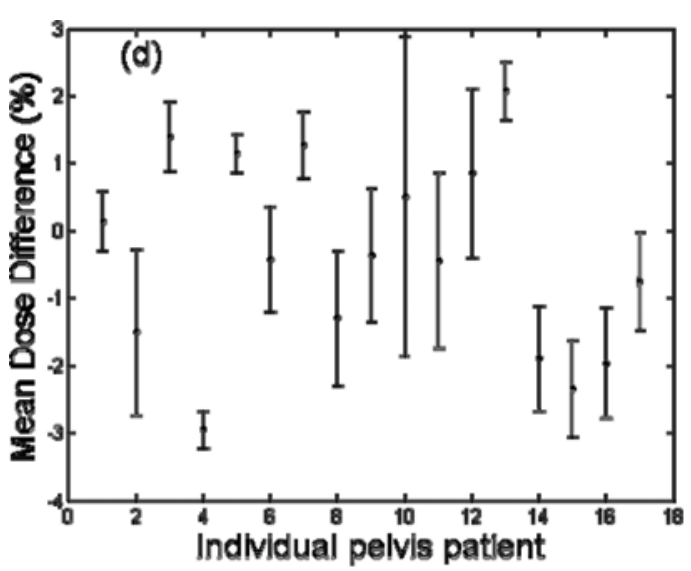

Figure 3: Mean random variation of dosimetric consequences with standard deviations for each patient over its treatment fractions.

Dose dif ferences were calculated with MU-Tomo with random shifts. Dosimetric consequences are shown in the following plots for (a) prostate patients, (b) head \& neck patients, (c) lung patients, (d) pelvis patients, and (e) brain patients. 


\begin{tabular}{|l|l|l|l|l|}
\hline Treatment site & Patient \# & Mean & Standard Deviation & Max \\
\hline Prostate & 30 & $0.51 \%$ & $1.55 \%$ & $3.74 \%$ \\
\hline Head \& Neck & 26 & $0.038 \%$ & $2.54 \%$ & $4.82 \%$ \\
\hline Lung & 18 & $0.12 \%$ & $1.86 \%$ & $-3.11 \%$ \\
\hline Pelvis & 17 & $-0.38 \%$ & $1.46 \%$ & $-5.65 \%$ \\
\hline Brain & 9 & $0.82 \%$ & $2.26 \%$ & $-3.58 \%$ \\
\hline
\end{tabular}

Table 4: Treatment site specif c random dosimetric difference result compared to the planned dose. Daily setup shifts were corrected by subtracting the systematic shifts.

\begin{tabular}{|l|l|l|l|l|l|}
\hline Case 1 & Case 2 & Case 3 & Case 4 & Case 5 \\
\hline$p<0.05$ & $p>0.05$ & $p>0.05$ & $p<0.05$ & $p>0.05$ & $p>0.05$ \\
\hline
\end{tabular}

Table 5: $\mathrm{P}$ values for all tests done. Case 1: Kruskal-W allis test among dif ferent patients for random dosimetric variations. Case 2: Kr uskal-Wallis test among dif ferent patients for systematic dosimetric variations. Case 3: Kruskal-W allis test among two groups of treatment sites for random dosim etric variations. Case 4: Kruskal-W allis test among two groups of treatment sites for systematic dosimetric variations. Case 5: Kruskal-W allis test among prostate, lung, and pelvis cancers for both random and systematic dosimetric variations. Case 6: Kruskal-Wallis test among head \& neck, and brain cancers for both random and systematic dosimetric variations.

\begin{tabular}{|l|l|l|l|l|}
\hline Pearson test & Prostate & Head\&neck & Lung & Pelvis \\
\hline Coeff cient 1 & 0.46 & 0.039 & -0.48 & -0.23 \\
\hline Coeff cient 2 & 0.34 & -0.016 & -0.017 & -0.67 \\
\hline
\end{tabular}

Table 6: Pearson correlation test between treatment fraction number (TFN) and dosimetric consequences. The Pearson correlation coeff cients are shown along treatment sites for both systematic (coeff cient 1) and random (coeff cient 2) dosimetric variations.

(Figure 3 here. Mean random dosimetric variation with standard deviations for each patient over its treatment fractions.)

Figure 3 (a) to (e) shows the mean random dosimetric variations with corresponding standard deviations of dosimetric consequences for all treatment fractions for prostate, head \& neck, lung, pelvis, and brain cancer patients. For each patient, a random dosimetric variation from a specific fraction was calculated by incorporating the random daily setup shift after systematic shift correction to the dose calculation software. The mean value is shown for each patient. The largest dose deviations due to the random shift occurred to head \& neck and brain patients. One was $5.57 \%$ dose deviation from a brain patient and the other was $-5.65 \%$ from a head \& neck patient.

(Table 4 here. Treatment site specific random dosimetric difference result compared to the planned dose.)

The treatment site specific dosimetric consequences reported in Table 4 are sorted into the five different groups. These results were corrected after systematic shift subtraction. For all five groups of patients, the averaged random dosimetric variations were all within $\pm 1 \%$. The largest two standard deviations for the treatment site specific random dosimetric variations occurred at the head $\&$ neck site with $2.54 \%$ and brain site with $2.26 \%$. This is consistent with the results from the patient specific random dosimetric variation.

According to the Kruskal-Wallis statistical test for the patient specific dosimetric consequences due to inter-fractional setup shifts, highly statistically significant differences among different patients were discovered $(\mathrm{p}<0.05)$ for all prostate, head \& neck, lung, pelvis, and brain cancer patients for random dosimetric variations. No significant differences were examined for systematic dosimetric variations from all patients and all treatment sites $(\mathrm{p}>0.05)$. The ANOVA statistical test of treatment site specific dosimetric consequences due to inter-fractional setup shifts between the body group and head \& neck and brain group showed no significant difference for the random dosimetric variations $(p>0.05)$. On the contrary, significant difference was found for systematic dosimetric variations between the two groups $(p<0.05)$. Furthermore, the Kruskal-Wallis test showed that there were no significant differences within the body group among prostate, lung, and pelvis cancer patients for both systematic and random dosimetric variations. No significant differences were examined between the head \& neck and brain cancer patients for both systematic and random dosimetric variations. The Pearson correlation statistical method found no strong correlations between the treatment fraction number and dosimetric consequences of patients with the same treatment site. After analysis, the Pearson correlation coefficients between the treatment fraction number and random dosimetric variations are $0.34,-0.016,-0.017,-0.35$, and -0.62 for prostate, head \& neck, lung, pelvis, and brain cancer sites, correspondingly. The Pearson correlation coefficients between the treatment fraction number and systematic dosimetric variations are $0.46,0.039,-0.48,-0.23$, and -0.67 for prostate, head \& neck, lung, pelvis, and brain cancer sites, correspondingly. Furthermore, systematic dosimetric variations were found significantly different from mean random dosimetric variations and systematic dosimetric variations were significantly larger than mean random dosimetric variations $(\mathrm{p}=0.00032)$.

(Figure 4 here. Benchmarked dose differences tweaked to nonshift (reference) dose differences for 100 patients.)

(Table 5 here. $\mathrm{p}$ values for all tests done).

(Table 6 here. Pearson correlation test between treatment fraction number (TFN) and dosimetric consequences).

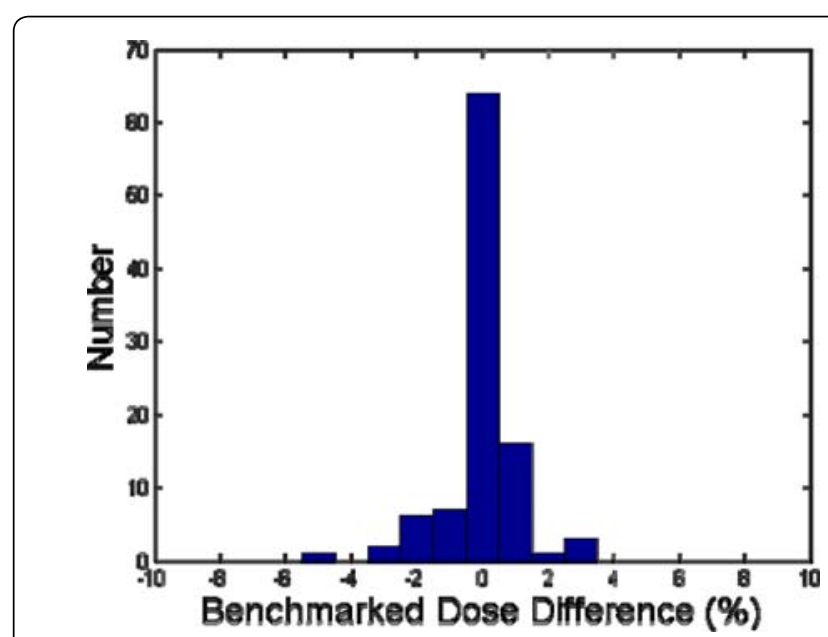

Figure 4: Benchmarked dose dif ferences show the overall dosimetric impact after systematic correction for each of the hundred patients. The benchmarked dose dif ference is de $f$ ned by subtracting the non-shift (reference) dose difference from the mean random dose difference. 
The benchmark dose differences from all one hundred patients are illustrated in Figure 4. For each patient, the benchmark dose difference denotes the averaged dosimetric impact away from planned dose after all treatment fractions. Ninety-nine percent of the case studies were within $\pm 3.5 \%$ of the dose difference with only one of them at $-5.39 \%$. The mean benchmark dose difference is almost zero $(-0.0053 \%)$ with a standard deviation of $1.11 \%$.

\section{Discussion}

This work has studied inter-fractional errors due to daily setup uncertainties for one hundred helical tomotherapy cases from five treatment sites with $M U$-Tomo. $M U$-Tomo provides a fast independent dose calculation for tomotherapy. Based on the commissioned dosimetric data from our tomotherapy unit, our method retrieves the dynamic information such as the MLC movement, gantry projections, and couch translations with specified pitch from specific patient treatment planning. These dynamics facilitate our dose calculation and provide good coupling to the daily patient setup shift.

The dosimetric consequences due to the inter-fractional motions were derived on the assumption that patients were rigid bodies. Thus, deformable activities inside a patient may cause more uncertainties for the registration. Since this work studied the interfractional nature and impact to the dose distribution, rigid body assumption is a simple way for the incorporation of the setup shift on coordinates, which may introduce calculation inaccuracy. More efforts are required to estimate the intra-fractional motions, which would be a good topic for an independent study.

The dosimetric consequences were classified into systematic and random dosimetric variations in our study. Systematic dosimetric variation was derived from the systematic shift for individual patients, and random dosimetric variation was derived from the random shifts after systematic correction for each treatment fraction. The reason to separate the study is due to the different features between the systematic and random dosimetric variations. The systematic dosimetric variation represents the mean overall effect of dosimetric consequences due to inter-fractional shifts, and the random dosimetric variation represents the specific fractionized effect of dosimetric consequences due to inter-fractional shifts. Our results show that systematic shifts generally cause more dose deviation than random daily shifts. The standard deviation was $3 \%$ and the mean value was $-0.91 \%$ for the systematic dosimetric variations of all patient plans. While the standard deviation was $1.9 \%$ and the mean value was $0.19 \%$ for the mean random dosimetric variations of all patient plans. This is reasonable, since the random daily setup shifts were calculated based on the systematic shifts and relatively less impact is expected between different fractions for each patient.

Based on the ANOVA analyses, patient specific dosimetric consequences were found to be significantly different for random dosimetric variations among all patients without significant difference for systematic dosimetric variations. While treatment site specific dosimetric consequences were found significantly different for systematic dosimetric variations and no significant difference for random dosimetric variations between the body group and the head $\&$ neck and brain group. Meanwhile, no significant difference was found among the body group and among the head \& neck and brain group, separately. This implies that there are significant variances among individual patients for random shifts, and systematic shifts impact the dosimetric consequences to different treatment sites more than individual patients. Individual patients may have their own characteristics in daily setup due to different anatomies and activities, so that individual registration may not be normally derived from a population of observations. On the other hand, treatment specific studies show different features in systematic dosimetric variations between groups with different internal motions and common agreement within each group. This indicates that the systematic shift impact is relatively treatment site related. Different treatment sites may exhibit different setup shifts and require different registration techniques and tolerances. Patients with different treatment sites indicate to group accordingly for dosimetric studies. Furthermore, the correlations between treatment fraction numbers and dosimetric consequences within patients were found not strong. The fraction number may not be an important factor in impacting the dose distribution during inter-fractional setups.

To estimate the averaged dosimetric impact from all fractions of each patient, the benchmark dose differences were calculated. Our results show that the averaged dose deviations are very small, and the distribution is around zero with a small standard deviation.

Limitations about this study include point dose comparison only and random sampling of the point inside the target. Those limitations may be overcome by comparing multi-point calculations from PTV or surrounding sensitive structures, two dimensional or three dimensional dose distributions in the future, such as using the adaptive treatment planning tool from TomoTherapy Inc. However, the current method has provided a good way to calculate the dose independently for tomotherapy modality in a short time. Different characteristics from systematic and random inter-fractional shifts show different dosimetric impacts on patient specific and treatment site specific plans. It is good to notice that head \& neck and brain patients, who have the small daily setup shifts due to immobilization equipments, may have large dosimetric variations. Plans on these two sites focus on the superficial or small regions. It is reasonable that they are sensitive to position shifts, and IGRT should be carefully managed for these patients. Additional efforts are required to get more accurate results.

\section{Conclusion}

A comprehensive study on dosimetric consequences due to interfractional setup shifts of one hundred helical tomotherapy patients from five different treatment sites was performed. The mean daily setup shift for each patient was quantified as the systematic shift. Daily setup shifts subtracting the systematic shift imitate the random shift for each treatment fraction. Systematic dosimetric variations are more related with treatment site specific patient plans. Random dosimetric variations are more related with specific patients. Systematic dosimetric variations were generally found to contribute more to dose deviations than random dosimetric variations. Dosimetric consequences are not significantly correlated with treatment fraction number. The overall dosimetric impacts to each patient are found to be small after the systematic shift correction.

\section{Acknowledgement}

This project is sponsored partially by Oncology Data Systems, Inc., Oklahoma City, OK, USA.

\section{References}

1. Balter JM, Brock KK, Lam KL, Tatro D, Dawson LA, et al. (2005) Evaluating the inf uence of s etup $u$ ncertainties o $n t$ reatment $p$ lanning $f$ or $f$ ocal I iver tumors. Int J Radiat Oncol Biol Phys 63: 610-614.

2. Broggi S, Cozzarini C, Fiorino C, Maggiulli E, Alongi F, et al. (2009) Modeling set-up e rror by d aily M VCT for prostate adjuvant treatment delivered in 20 fractions: Implications for the assessment of the optimal correction strategies. Radiother Oncol 93: 246-252. 
3. Broggi S, Di M uzio N, F iorino C, C ozzarini C, C attaneo G M, et al. (2007) Prostate tracking through daily MVCT-guided tomotherapy: Reduced motion by careful emptying of the rectum. Radiother Oncol 84: 393

4. Fiorino C, D i M uzio N, B roggi S, C ozzarini C, M aggiulli E, e t a I. (2007) Evidence of limited motion of the prostate by c arefully emptying the rectum as assessed by d aily MVCT image guidance with helical tomotherapy. Int J Rad Oncol 71: 611-617.

5. Fu WH, Yang Y, Li X, Heron DE, Huq MS, et al. (2006) Dosimetric effects of patient rotational setup errors on prostate IMRT treatments. Phys Med Biol 51: $5321-5331$

6. Gibbons JP, S mith K, Cheek D, Rosen I (2009) Independent calculation of dose from a helical TomoTherapy unit. J Appl Clin Med Phys 10: 2772.

7. Goddu S M, Yaddanapudi S, Pe chenaya O L, C haudhari S R, K lein E E, e al. (2009) D osimetric c onsequences of uncorrected s etup e rrors in helical Tomotherapy treatments of breast-cancer patients. Radiother Oncol 93: 6470.

8. ICRU Report 29 (1978) Dose specif cations f or $r$ eporting ex ternal b eam therapy with photons and electrons. Bethesda, MD, International Committee on Radiation Units and Measurements.

9. Kalz J, Sterzing F, Schubert K, Sroka-Perez G, Debus J, et al. (2009) Dosimetric Comparison of Image Guidance by Megavoltage Computed Tomography ve rsus $B$ one $A$ lignment $f$ or $P$ rostate $C$ ancer $R$ adiotherapy. Strahlenther Onkol 185: 241-247.

10. Keall PJ, Mageras GS, Balter JM, Emery RS, Forster KM, et al. (2006) The management of respiratory motion in radiation oncology report of AAPM Task Group 76. Med Phys 33: 3874-3900.

11. Landoni V, S aracino B, M arzi S, G allucci M, Pe trongari M G, et a I. (2006) A study of the effect of setup e rrors and organ m otion on prostate cancer treatment with IMRT. Int J Radiat Oncol Biol Phys 65: 587-594.

12. Lee C, L angen KM, Lu W, H aimerl J, S chnarr E, et a I. (2008) Evaluation of geome tric $\mathrm{c}$ hanges of parot id glan ds dur ing head and nec $\mathrm{k} \mathrm{c}$ ance radiotherapy $\mathrm{u}$ sing $\mathrm{d}$ aily $\mathrm{M}$ VCT a nd a utomatic $\mathrm{d}$ eformable $\mathrm{r}$ egistration. Radiother Oncol 89: 81-88.

13. Lefkopoulos D , Fe rreira I, I sambert A, L e Pe choux C , M ornex F ( 2007) [Present a nd $f$ uture of $\mathrm{t}$ he I mage $\mathrm{G}$ uided R adiotherapy (I GRT) a nd $\mathrm{i}$ ts applications in lung cancer treatment.] Cancer Radiotherapie 11: 23-31.

14. Li XA, Qi XS, Pitterle M, Kalakota K, Mueller K, et al. (2007) Inter-fractional variations in patient setup and anatomic change assessed by daily CT from helical tomotherapy. Int J Radiat Oncol Biol Phys 68: 581-591.

15. Lin L, Shi CY, Eng T, Swanson G, Fuss M, et al. (2009) Evaluation of Interfractional Se tup Shif ts for Sit e-specif $\mathrm{c} \mathrm{H}$ elical T omotherapy $\mathrm{T}$ reatments. Technol Cancer Res Treat 8: 115-122.

16. Low DA, Zhu XR, Purdy JA, Soderstrom S (1997) The inf uence of angular misalignment on $\mathrm{x}$ xed-portal intensity modulated radiation therapy. Med Phys 24: $1123-1139$
17. Lu W, Chen M, Ruchala KJ, Chen Q, Langen KM, et al. (2009) Real-time motion-adaptive-optimization ( MAO) in TomoTherapy. P hys M ed Biol 54 4373-4398

18. Mackie TR (2006) History of tomotherapy. Phys Med Biol 51: R427-R453.

19. Mackie T R, K apatoes J, R uchala K, L u W G, Wu C , et a I. (2003) I mage guidance for precise conformal radiotherapy. Int J Rad Oncol 56: 89-105.

20. Manning M, Wu Q , C ardinale R M, M ohan R, L auve A D, et al. (2001) The effect of setup uncertainty on normal tissue sparing with IMRT for head-andneck cancer. Int J Radiat Oncol Biol Phys 51: 1400-1409.

21. Ngwa W, Meeks SL, Kupelian PA, Schnarr E, Langen KM (2009) Validation of a computational method for assessing the impact of intra-fraction motion on helical tomotherapy plans. Phys Med Biol 54: 6611-6621.

22. Perkins CL, Fox T, Elder E, Kooby DA, Staley CA, et al. (2006) Image-guided radiation therapy (IGRT) in gastrointestinal tumors. Jop 7: 372-381.

23. Ryan D, Rivest C, Riauka TA, Murtha AD, Fallone BG (2009) Prostate positioning e rrors a ssociated with two a utomatic registration b ased image guidance strategies. J Appl Clin Med Phys 10: 165-176.

24. Sawant A, Venkat R, Srivastava V, Carlson D, Povzner S, et al. (2008) Management of $\mathrm{t}$ hree-dimensional i ntrafraction $\mathrm{m}$ otion $\mathrm{t}$ hrough $\mathrm{r}$ eal-time DMLC tracking. Med Phys 35: 2050-2061.

25. Schubert LK, Westerly DC, Tomé WA, Mehta MP, Soisson ET, et al. (2009) A C omprehensive A ssessment by $T$ umor $S$ ite o $\mathrm{f} P$ a tient $S$ etup $\mathrm{u}$ sing Daily $\mathrm{M}$ VCT I maging $\mathrm{f}$ rom $\mathrm{O}$ ver $\mathrm{T}$ hree $\mathrm{T}$ housand $\mathrm{E}$ ight $\mathrm{H}$ undred $\mathrm{H}$ elical Tomotherapy Treatments. Int J Radiat Oncol Biol Phys. 73: 1260-1269.

26. Sheng K, Chow J, Hunter G, Larner JM, Read PW (2008) Is daily CT image guidance necessary for nasal c avity and nasopharyngeal radiotherapy: an investigation based on helical tomotherapy. J Appl Clin Med Phys 9: 2686

27. Siebers J V, Ke all P J, Wu Q W, W illiamson J F, S chmidt-Ullrich R K (2005) Effect of patient setup errors on simultaneously integrated boost head and neck IMRT treatment plans. Int J Rad Oncol Biol Phys 63: 422-433.

28. Stutzel J, Oelfke U, Nill S (2008) A quantitative image quality comparison of four different image guided radiotherapy devices. Radiother Oncol 86: 20-24.

29. Su FC, Shi CY, Crownover R, Swanson G, Papanikolaou N (2008) Dosimetric impacts of $g$ antry a ngle $m$ isalignment on p rostate $c$ ancer t reatment $u$ sing helical tomotherapy. Technol Cancer Res Treat 7: 287-293.

30. Xing L, Lin ZX, Donaldson SS, Le QT, Tate D, et al. (2000) Dosimetric effects of pat ient displac ement and collimator and gan try angle misalignment on intensity modulated radiation therapy. Radiother Oncol 56: 97-108.

31. Yan D, Lockman D, Martinez A, Wong J, Brabbins D, et al. (2005) Computed tomography g uided management of interfractional patient va riation. Semin Radiat Oncol 15: 168-179.

32. Zhou J, Uhl B, Dewit K, Young M, Taylor B, et al. (2010) Analysis of daily setup variation with tomotherapy megavoltage computed tomography. Med Dosim 35: $31-37$. 